\title{
Evaluating Display Modalities Using a Mixed Reality Game
}

\author{
Hitesh Nidhi Sharma, Zachary O. Toups, Igor Dolgov, Andruid Kerne, Ajit Jain ${ }^{3}$ \\ ${ }^{1}$ Play \& Interactive Experiences for Learning Lab, Dept. Computer Science, New Mexico State University \\ ${ }^{2}$ Perception, Action, \& Cognition in Mediated Artificial \& Natural Environments Lab, Dept. Psychology, \\ New Mexico State University \\ ${ }^{3}$ Interface Ecology Lab, Dept. Computer Science \& Engineering, Texas A\&M University \\ hitesharmaa@gmail.com, z@cs.nmsu.edu, id@nmsu.edu, andruid@ecologylab.net, ajit@ecologylab.net
}
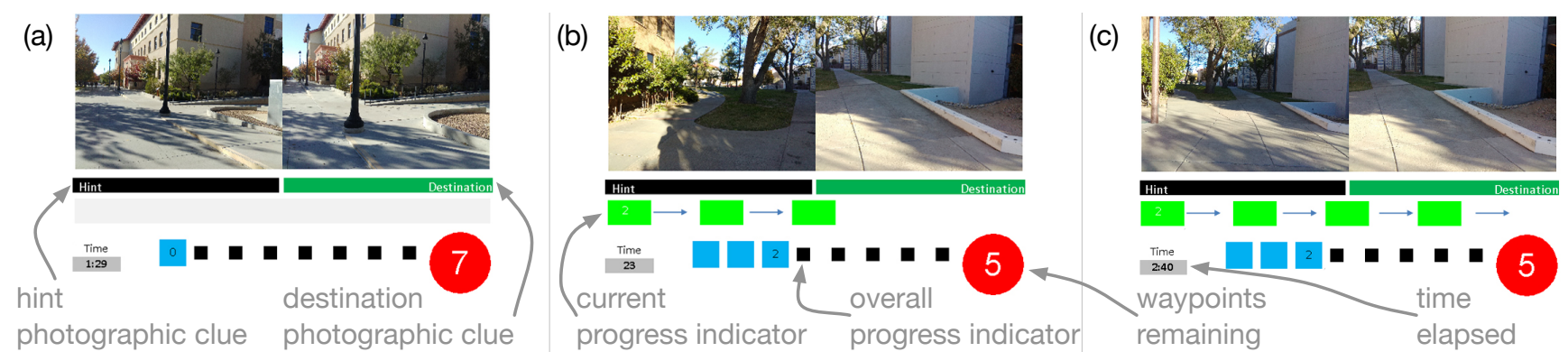

Figure 1. The PhotoNav game user interface, showing player progress along a course, guided by a hint photographic clue to a destination photographic clue. UI components populate the bottom. The current progress indicator shows the advancement of a player toward the current waypoint. The overall progress indicator shows progression along all the waypoints. In: (a) the player starts at the first waypoint (7 waypoints remain), (b) the player moves from 2 nd to the 3 rd waypoint ( 5 waypoints remain), (c) the player continuing progression nearing the current destination (5 waypoints remain).

\begin{abstract}
We design and study a mixed reality game, PhotoNav, to investigate wearable computing display modalities. We study game play, varying display modality: head-mounted, handheld, and wrist-worn. PhotoNav requires the player to split attention between the physical world and display by using geotagged photographs as clues for navigation. Our results showed that participants using the head-mounted display exhibited poor performance in one of the courses and that players preferred handheld and wrist-worn displays, likely due to their familiarity with them. We derive design implications from the study and discuss problems faced in designing mixed reality games and interfaces that require users to split their attention.
\end{abstract}

\section{ACM Classification Keywords}

H.5.1. Information Interfaces and Presentation (e.g. HCI): Multimedia Information Systems: Artificial, augmented, and virtual realities

\section{Author Keywords \\ Display modalities; mixed reality; wearable; handheld display; head-mounted display; wrist-worn display.}

Permission to make digital or hard copies of all or part of this work for personal or classroom use is granted without fee provided that copies are not made or distributed for profit or commercial advantage and that copies bear this notice and the full citation on the first page. Copyrights for components of this work owned by others than the author(s) must be honored. Abstracting with credit is permitted. To copy otherwise, or republish, to post on servers or to redistribute to lists, requires prior specific permission and/or a fee. Request permissions from permissions@ acm.org.

CHI PLAY '16, October 16 - 19, 2016, Austin, TX, USA

(C) 2016 Copyright held by the owner/author(s). Publication rights licensed to ACM. ISBN 978-1-4503-4456-2/16/10 . \$ \$15.00

DOI: http : //dx . doi .org/10.1145/2967934. 2968090

\section{INTRODUCTION}

Handheld displays, in the form of smartphones and gaming devices, are common in many parts of the world; wrist-worn and head-mounted displays (HMDs) have begun to proliferate. Open questions remain about the ability of wearable computers to enhance performance, improve situation awareness, and reduce mental workload. Already, cases where users split their attention between the physical world and digital data arise in urban contexts, where individuals attend to devices and the environment simultaneously (e.g., reading email and walking). New use cases for wearable computers emerge beyond mundane scenarios, such as disaster responders with wearables for local sensor telemetry and incoming warnings displayed on facemasks or soldiers with information displays built into helmets.

To address these use cases and study the best designs for wearables moving forward, the present research tests wearable computer designs using mixed reality games to better understand how they impact human performance in the physical world. We develop PhotoNav, a game that engages players both in an information device and in the physical world. We use this game to investigate display modality: the relationship between the display and the user's body. We test HMD, handheld, and wrist-worn display modalities which act as proxies for devices available on the market (e.g., HMD mobile devices, smartwatches, and smartphones, respectively). We expect HMD to perform better due to shorter shift in attention.

In our mixed reality game, a player chooses a path through physical environments, seeking out waypoints using photo- 
graphic clues. The game UI shows two photographs: one of the destination and one hint taken nearby, the vantage point of which will advance the user toward the destination waypoint. The game is designed such that the player must use the photographs, and not other UI components, to traverse the environment. The UI components have been designed to provide useful status information while making them useless for direct navigation, forcing reliance on the photographic clues.

A key component of the game's UI design, in order to simulate wearable computing use cases, is to split the player's attention between device and the physical world. The photographic clues provide a tension: they are necessary to determine where to go, but draw attention away from scanning the physical world in search of the next clue. The current research builds on our prior pilot study [38].

We proceed to connect together background. We then describe our custom wearable computer, used for the present game, as well as game mechanics and design of PhotoNav. We describe a user study, discuss implications for design, and present conclusions.

\section{BACKGROUND}

The background for this project combines research into wearable computing, game design, mixed reality, splitting attention, and navigation.

\section{Mobile and Wearable Computing}

Little scholarly attention has been paid to non-handheld mobile display modalities, likely due to the lack of practical wearable designs $[8,11,20]$, until recently. While smartphones and tablets have become commonplace in many countries, everyday wearable technologies are just beginning to proliferate through wrist-worn displays (e.g. Pebble, Apple Watch, Android Wear) and HMDs (Google Glass, Microsoft HoloLens, Meta Pro, Sony SmartEyeglass).

Wearable computers distribute computing technology across the body $[26,41]$. While we have seen a recent increase in the commercial availability of wearable technologies, the present research uses a modular, backpack wearable, that serves as a development platform for multiple mixed reality scenarios with a minimal set of hardware. Wearables incorporating general purpose computing, location and direction sensors, head tracking, and/or HMDs, were pioneered by Mann, Starner, Feiner, MacIntyre, and others for the applications of personal, day-to-day augmentation [40], data collection [27], locationbased information gathering [15], and/or gaming [30,45].

Although HMDs have been compared to desktop and largescale displays [37,39,42], there is a knowledge gap in wearable contexts. HMDs enable the user to access information directly in the visual field, but users must merge the visual field and computer display $[49,50]$. Moreover, social pressures may impact appropriateness of device use [34].

Two prior empirical studies compared display modalities $[47,52]$. Yeh et al.'s simulated mission study [52] showed that participants detected targets better using a handheld navigation aid over a helmet-mounted one; display clutter diminished performance. Vadas et al. [47] undertook a mobile in-the-laboratory [1] study in which participants used either an HMD, a handheld e-ink display, or a backlit handheld display to read while walking. The HMD fared worst for walking performance, but not reading comprehension, due to participants' split attention.

Numerous other studies have compared pilots' performance using head-up and head-down displays [14]. Unlike HMDs, head-up displays (HUDs) are fixed inside the cockpit/cabin while HMDs effectively track users' head movements. A headdown display is analogous to a handheld or wrist-worn one, except that the handheld or wrist-worn display may be moved, where the head-down display is fixed.

\section{Game Design}

Game play fundamentally involves making decisions. A game mechanic is a designed set of rules through which a player makes a choice, advancing toward an outcome [36]. Rules constrain play, rendering it meaningful. The core mechanics are the set of game mechanics engaged repeatedly.

\section{Mixed Reality}

Systems that connect physical and virtual reality in some meaningful way through sensors and databases, are mixed realities $[6,7,9,29]$. These range from augmented reality, in which 3D imagery is integrated with a perspective on the physical world, as with most HUDs, to augmented virtuality, in which physical-world artifacts and spaces are integrated into a virtual world. Mixed reality systems offer the opportunity to create embodied interfaces [12], in which participants create meaning through hybrid digital / physical experiences.

The present research involves mobile mixed reality, which provides the user a location-specific experience $[3,15,16]$. Users are tracked with GPS-based location sensors $[10,16,53]$, which drive the player's experience of the virtual world. The user's physical-world location is mapped to a virtual-world location isomorphically.

\section{Splitting Attention}

Many tasks require users to split attention when they need to focus on the physical world and a display interface simultaneously (e.g., reading email while walking, navigating using a map). As wrist-worn displays and HMDs also help to accomplish similar tasks, we investigate which display modality works best when users need to split their attention. Trevisan et al. showed that when real and virtual worlds interact, designers must focus a user's attention on one or the other [46], pointing out that the ability to focus is affected by UI design. We investigate intentional splitting of attention as a part of gameplay, making decisions about where to focus a game mechanic.

\section{Navigation}

In a prior study [31], a pedestrian navigation assistant was developed to compare view-based wayfinding using pictures to incidental learning using map-based wayfinding; the study showed that users developed good knowledge of routes while using both map-based and view-based navigation assistance. Beeharee et al. [5] found that geo-annotated photographs aid pedestrians in following routes. They augmented photographs 
(a)

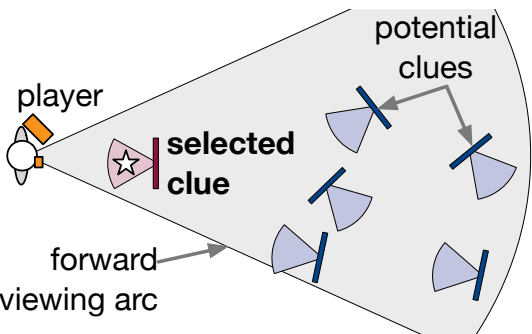

(b)

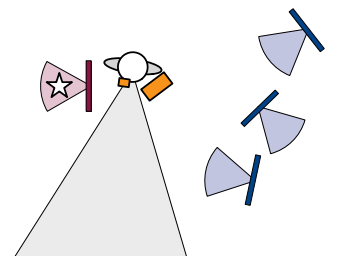

(c)

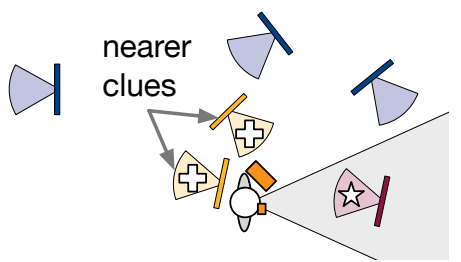

Figure 2. Top-down view of photographic clue selection algorithm as a player progresses through a space rich with clues. Potential clues are shown with their reverse orientation arc (so standing within a clue's arc suggests one could see the physical world the photograph represents). Clues that are closest and whose reverse orientation arc align with the orientation of the player, as determined by the head-mounted compass, are selected. In (a), the simplest case, where the nearest clue is in view; in (b) a nearby clue outside of view is selected because no clues are in view; in (c) an image that should be visible is selected over other, nearer clues.

with direction cues to minimize navigational ambiguities [4]. May et al. [28] studied requirements of pedestrians within an urban context and identified that providing information at key decision points and confirming correct decision are useful mobile navigation features. In the present study, we provide navigational clues through photographs.

\section{PHOTONAV DESIGN}

We designed PhotoNav to evaluate the utility of display modalities in demanding situations by purposefully splitting the player's attention between physical world and device. The physical world forms a component of the digital game. We constructed the game to provide minimal information to the player for decision making, using photographic clues to guide players through a course. The game was designed to be playable with any display modality.

\section{Wearable Computer}

We developed a modular wearable computer that combines computing, tracking, feedback, and minimal direct user interaction; Figures 3 and 4 show the wearable. We use this wearable, rather than existing commercial solutions, due to its flexibility and ease-of-development. We use GPS to obtain player location, while a forehead-mounted compass provides head tracking. The wearable incorporates an HMD and a small wired display, which can either be handheld or strapped onto an arm to act as a wrist-worn display. While modern smartwatch interfaces are smaller in size, given the nature of the study, the same wired display was used as a wrist-worn display as it would be difficult to compare two different user interfaces. Feedback is provided to the user using the active display modality. Details are provided in the apparatus section.

\section{Core Mechanics}

PhotoNav is a location-aware game in which a player moves through a physical environment with the goal of finding hidden waypoints using photographic clues. The core mechan$i c s$, about which players make decisions, are freely moving through the environment, paying activating the display, exploring, and seeking out the clues provided.

Each round of PhotoNav is played on a course (several of which are run for a study). Courses consist of a sequence of waypoints and a collection of photographic clues: geotagged and oriented photographs. Hint photographic clues are algorithmically selected at runtime such that, if the player advances toward the photographed location, s/he will be closer to the destination. Each waypoint has a single destination photographic clue. As the player moves, system provides new hints. Players accrue score based on finding each waypoint quickly.

\section{Game Interface and Photographic Clues}

The game interface is designed to provide only sufficient information for the player to make informed decisions about where to move. The PhotoNav UI is minimal (Figure 1), the majority of it consists of photographic clues. As the player moves, the hint photographic clue updates. After finding the destination, the waypoint is updated and a new destination photographic clue is shown.

The game program selects clues based on the player's likelihood of seeing them, using a photographic clue selection algorithm (Figure 2). The algorithm uses two arcs: the forward viewing arc of the player, calculated from GPS location and compass orientation, and the viewable arc of each photograph, calculated from the photograph's geotag and inverse orientation. The selection criteria assume that a person in the viewable arc of the photograph, looking in the direction the photo was taken, will be able to see the photographed scene. To make selection more robust, PhotoNav will pick the nearest image if the player is not within any photograph's viewable arc. The maximum distance between the player and an image is fixed at 30 meters, and the view arcs are all 60 degrees. Photographic clue images are taken in advance of the game, during the same season as play, to minimize the differences between photographs and the physical world.

\section{Feedback}

The game UI provides supporting contextual information, including two progress indicators; Figure 1 shows the interface in a variety of progression points. The current progress indicator shows the progress of a player towards the current waypoint at a low resolution (only five states). The coarsegrained nature of the current progress indicator prevents its use as the sole means of navigation.

The display also includes an overall progress indicator at the bottom of the screen that shows total progress of the player. The progress indicator provides a series of blocks, as well as a single numerical value showing the remaining waypoints. 
These UI cues are minimal and obfuscated: as player progresses, hint photographic clues change. The feedback does not tell the player which direction to move; the player needs to make a decision based on the surrounding and clues.

\section{Player Input}

The player's direction and location are constantly monitored by the PhotoNav software to maintain and progress the game state. The player does not need to provide direct input to the game to play, but a handheld chording keyboard (Figure 3 right) is used to activate the display and allow the player to indicate that $\mathrm{s} / \mathrm{he}$ is already familiar with the destination.

The player can activate the display by holding down a button on the chording keyboard; otherwise, the display is blank. This requires players to explicitly indicate when they need to see the display; we use this as a proxy for the player's attention. By determining the total time players activate the display, we aim to find out how often players use the display or how it affects player performance.

For data-collection purposes, a familiarity button allows players to mark the current section of the course as familiar if they can reach the destination without hints. When the button is pressed, a countdown timer is used to confirm familiarity. If the button is held for 10 seconds, the systems registers player's familiarity with the immediate waypoint.

\section{Supporting Systems and Implementation}

The PhotoNav system is comprised of three components: the PhotoNav game itself, the NavCurator support application, and the PhotoNav Capture support application. While the PhotoNav game is our focus, the NavCurator and PhotoNav Capture applications were used to develop game courses and collect images for PhotoNav.

\section{PhotoNav Game Implementation Details}

PhotoNav is built in Java and runs on the wearable computer using a single active display modality (any other displays are blank). The hardware implementation drives the game resolution, which uses the maximum resolution of the HMD: $640 \times 480$ pixels. As the player starts the game, an interface is shown that provides a clue and a destination, both of which are images that are shown on the screen as shown in Figure 1.

The waypoints that make up each course are stored in an XML file, which references a collection of geotagged photographs. The XML file is generated by the experimenter using NavCurator. The S.IM.PL Libraries [17, 18] were used for de-/serialization to create, manage, and read the XML configuration files. The file only contains information about the waypoints, the clue and destination photographic hints are determined by the PhotoNav application at runtime.

PhotoNav can be run in two different modes: game mode and simulation mode. In the game mode, a player puts on a wearable computer and moves through real physical locations to explore the game. In simulation mode, PhotoNav uses Google Earth ${ }^{1}$ as a GPS emulator. The location data is then sent to PhotoNav application, which updates the images based

\footnotetext{
${ }^{1}$ https://www . google. com/earth/
}

on the acquired location data. This provides for an efficient way to test PhotoNav without having to be at real locations.

\section{NavCurator Support Application}

NavCurator is a Java application developed to create courses for PhotoNav. Users import geotagged images, markers of which are overlaid on top of aerial photographs. NavCurator is built on the NASA World Wind Platform ${ }^{2}$, which displays aerial photographs and satellite imagery of the globe. The imported images are plotted with their image direction. Users draw courses and the PhotoNav clue-selection algorithm identifies which images apply to the course. The created course can then be packaged up and exported to PhotoNav.

\section{PhotoNav Capture Support Application}

PhotoNav Capture is an Android application developed to accurately capture geotagged images and supply the player with enough context data to support creating courses in the $\mathrm{NavCu}-$ rator. Capture utilizes the in-built compass and GPS sensor of a tablet to tag images with location and direction information. It shows the player a map with photograph markers, so that the player can determine if enough photographs have been taken to cover an area. The images are saved in the tablet's internal memory and are transferred to a computer for NavCurator to plot and export PhotoNav-usable courses.

\section{METHODS}

The PhotoNav experiment was conducted with two conditions: display modality and course. Courses were specially designed sequences of waypoints that players needed to visit in order. Display modality was varied quasi-randomly among participants, but courses were always run in the same order owing to their geographic locations (Course A ended near Course B, which ended near Course C; see Figure 5 (optimal paths) to see the course layouts and how they connect). If courses were run out-of-order, participants would have had to walk through potentially future courses on the way to a course.

\section{Apparatus}

The wearable computer (Figures 3,4$)$ provides computation via a back-mounted notebook computer, tracking through a suite of sensors, and feedback through an HMD or handheld display (the latter of which may be used as a wrist-worn display). The wearable is built into a lightweight frame custom developed by Mystery Ranch ${ }^{3}$. Attached to the lower-back of the pack was a Dell Inspiron Mini 9910 notebook, (Windows XP Operating System, $1 \mathrm{GHz}$ Intel Atom processor, $1 \mathrm{~GB}$ RAM, 5 GB hard drive).

While the wearable has other sensors built-in, PhotoNav used a GPS and compass, both of which were head-mounted on the HMD mount. The GPS receiver was a Qstarz BT-Q818XT $10 \mathrm{~Hz}$ Bluetooth GPS receiver, connected to the computer via Bluetooth. An OceanServer OS5000-S digital compass was used for head tracking, connected to the notebook via a USB cable bound to the HMD cables. A Twiddler 3 chording keyboard / mouse is used as an input device for the game. The Twiddler is connected to the notebook computer via Bluetooth.

\footnotetext{
$2_{\text {http: //worldwind . arc . nasa . gov/java/ }}$

${ }^{3}$ http: //www . mysteryranch. com
} 


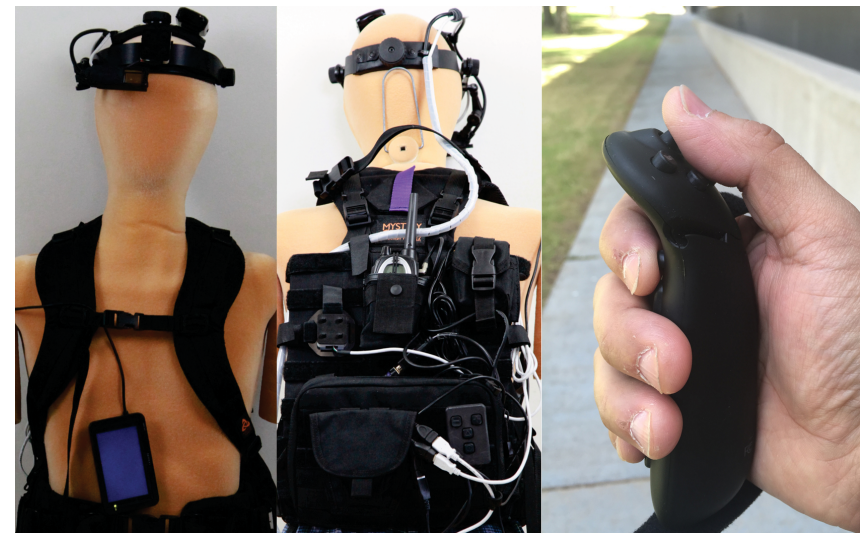

Figure 3. Front (left) and back (middle) views of the wearable computer. The wearable consists of: front: a monocular, see-through HMD with compass and GPS attached and a tethered handheld display; back: interconnects and a notebook computer. View of the chording keyboard, Twiddler (right): players used the thumb to record familiarity and the forefinger to bring up the interface.

\section{Display Modalities}

The wearable includes two displays to cover the three display modalities: an HMD and an external monitor that can be handheld or wrist-worn. The handheld device was a 4.3-inch Century LCD-4300U LCD display connected as an external monitor via a USB port to the notebook computer. The same LCD display was strapped to the hand of the participant to function it as a wrist-worn display as shown in Figure 4.

When we began developing the wearable computer in 2009, we considered a number of displays for the HMD modality. Tested systems included the Icuiti 920M, Vuzix Tac Eye, i-Port EX, and Liteye LE-750A; more recently, we tested a Google Glass Explorer Edition and Sony Smart Eyeglasses. The Liteye was chosen due to its ability to be used with corrective lenses, its extensive range of focus, and its ability to have the display placed in flexible locations. The display is monocular and see-through with a polarizing filter to reduce glare from the sun and adjustable brightness levels.

\section{Display Modality Conditions}

Three display modalities represented the three conditions in the study. In all conditions, the configuration of the wearable was held the same, with the appropriate display activated. In this way, we are not measuring the ergonomic impact of components of the wearable.

\section{Course Design}

Before taking the photographs, it was necessary to design the courses. There are many factors to be taken in consideration while choosing an outdoor space for any mixed reality system, especially player safety and the affordances [32] of the environment. We ensured a high density of photographic hints to keep players engaged and provide adequate feedback. We designed three courses which we expected to be unfamiliar to players.

All courses were developed with the following characteristics:

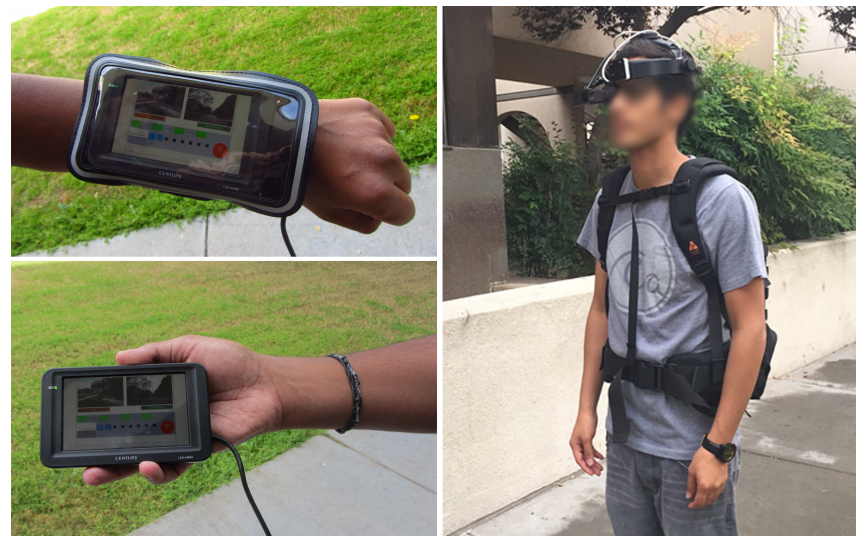

Figure 4. A player putting on a wrist-worn display (top left), player using a handheld display (bottom left). A participant putting on the HMD and wearable computer (right).

- were in pedestrian-safe areas;

- primarily featured open overhead space to avoid urbancanyon effects and/or other GPS interference [10,53];

- were selected from the New Mexico State University (NMSU) campus;

- feature seven waypoints (including the starting point); and

- contain at least three turns of approximately $90^{\circ}$.

The individual characteristics of the courses were as follows:

- Course A: 334 meters, 304 photographs, 3 turns. Passed near the following landmarks: Foster Hall, Young Hall, Hardman \& Jacobs Undergraduate Learning Center and Domenici Hall.

- Course B: 345 meters, 290 photographs, 4 turns. Passed near the following landmarks: Business Complex, Aggie Memorial Tower, Dove Hall, Hardman \& Jacobs Undergraduate Learning Center, and parking lot 11.

- Course C: 259 meters, 200 photographs, 4 turns. Passed near the following landmarks: Clara Belle Williams Hall, CHSS Annex, Speech Center.

Figure 5 (optimal paths) shows the layout of the courses, including the turns and how they connect in sequence.

As players rely on photographic clues to reach the waypoints, PhotoNav Capture was used to take pictures. The photos were captured such that there were about one photo per two meters, providing sufficient density to have hint photographic clues on and around the course.

\section{Subject Recruitment and Study Protocol}

The research protocol was approved by the NMSU IRB (protocol \#12948). Participants from NMSU were invited via flyers and emails to participate. Participants were not compensated. 

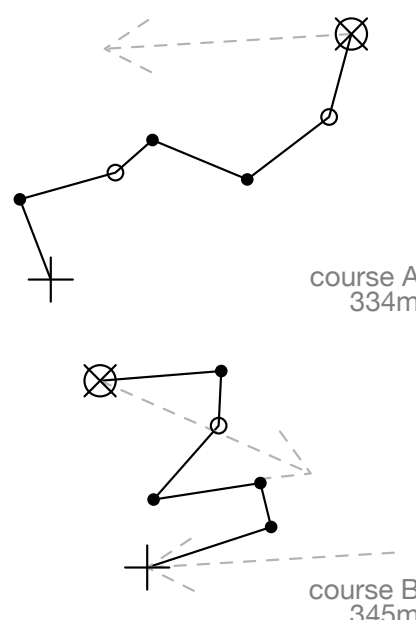

$345 \mathrm{~m}$

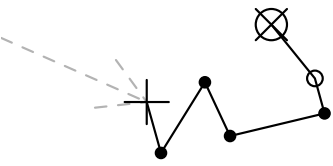

course C
$259 m$

(optimal paths)
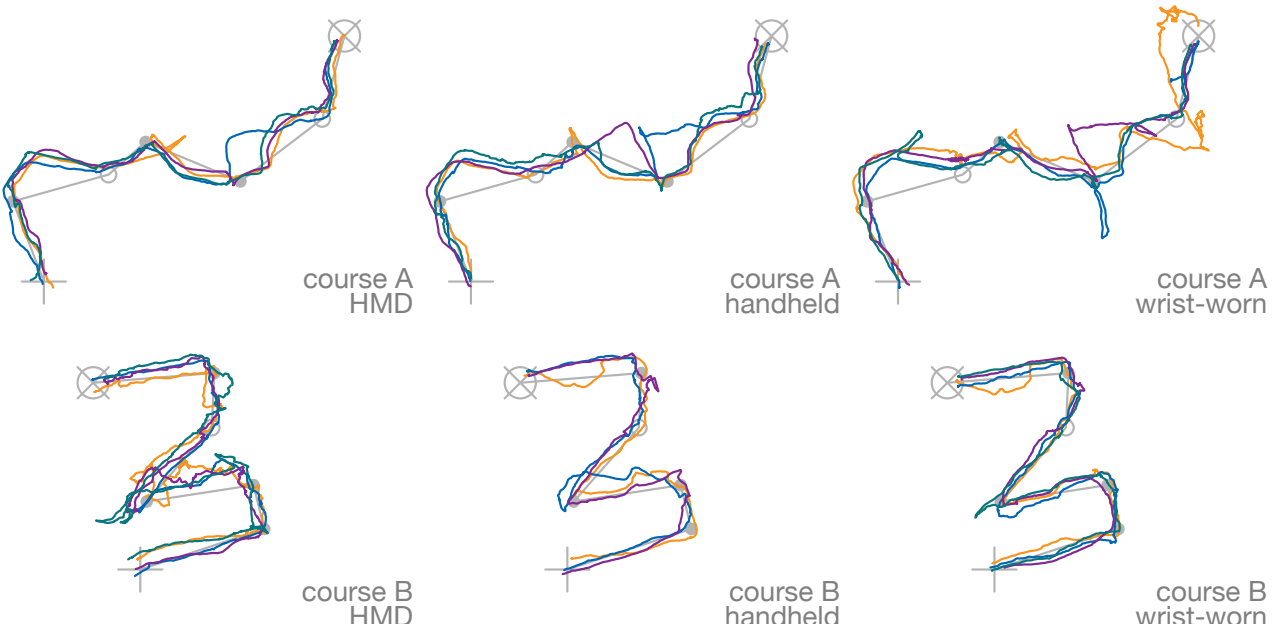

HMD

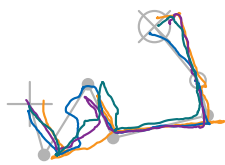

course C

(HMD condition)
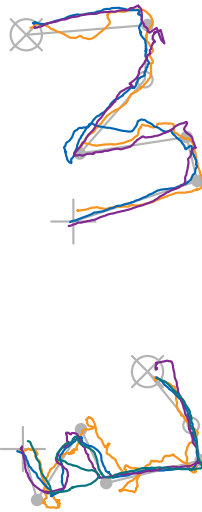

course B
handheld

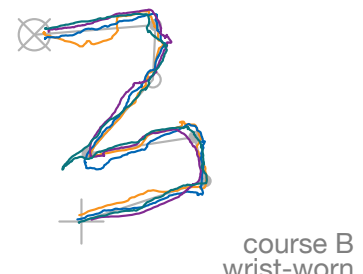

wrist-worn

Figure 5. A visualization of the optimal paths and actual paths taken in the user study. Courses are arrayed vertically (A-C); optimal paths are in the leftmost column with display modalities in the right three. Starting points are marked with a plus $(+)$, end points marked with a circle-X $(\otimes)$. Waypoints are indicated as filled circles for turns of approximately $90^{\circ}$; open circles for others. For the optimal paths, gray arrows indicate how courses connect together in a sequence. Colors are not mapped to data, only for distinguishing traversed paths.

As PhotoNav is played outdoors, players were first invited to a computer lab where participants provided informed consent. A video was then shown to introduce them to the game and its rules. The video explained the game interface, game mechanics, and instructions to operate the chording keyboard and how to focus on the HMD. We chose to use a video introduction, rather than tutorial, to avoid biasing participants toward a particular modality. The use of a video ensured that participants did not gain actual experience using the system prior to playing the game. The participants were then asked to complete a demographics and a navigation questionnaire. The demographics questionnaire consisted of age, gender, education. The navigation questionnaire addressed experience navigating, familiarity with GPS devices, and mixed reality games. It captured participants' navigation preferences and self-rating of prior navigation experiences using GPS and maps: the usage context (e.g. car, pedestrian).

\section{Data Collection}

During the user study, an XML-based log file recorded participants' game data, capturing game state, sensor state, and interaction state during each play. For game state, the log file recorded participant's time to reach each waypoint and current game progress. Sensor state consisted of GPS and compass data, which were taken at 1Hz: GPS coordinates, GPS health data (e.g., dilution of precision, number of satellites tracked), and compass orientation. We recorded the duration of time the UI was active (i.e., the player held the UI activate button on the Twiddler) and participants' indication of familiarity with the destination waypoints (i.e., when the player held the familiarity button for 10 seconds, the destination was marked familiar)

We asked the participants to fill out multiple ordinal questions after each session with each display modality. The Likert-scale questions we analyze for the present phase of research are the following:

1. How easy / difficult was it to view photographic clue on the display?

[“very easy”, "easy”, "neutral”, "difficult", "very difficult”];

2. Rate the display in matching images to the outside world: ["very easy", "easy", "neutral", "difficult", "very difficult"];

3. Rate the display in ease of viewing the interface: ["very easy", "easy", "neutral", "difficult", "very difficult"];

4. How safe did you feel while using the device and walking along the way points?

["very safe", "safe", "neutral", "unsafe", "very unsafe"];

5. Before the study, how familiar were you with the course? ["very unfamiliar", "unfamiliar","neutral”, "familiar", "very familiar"]; 
6. Where did you focus the most in the interface?

[Multiple allowed: "destination", "hint", "time", "overall progress", "current progress"].

We also asked participants how enjoyable they found the game.

\section{EXPERIMENT EXECUTION}

In fall and winter of 2015, a study was conducted on PhotoNav with 12 volunteers ( 5 female) from the student population of NMSU. Participants' ages ranged from 24-35 years old $(M=27, S D=3.4)$. All participants were graduate students. All participants had used smartphones; none reported prior use of HMD or smartwatch. The study used a within-subjects design with display condition (HMD, handheld display, wristworn display) counterbalanced.

The wearable itself is unchanged for the conditions, only the active display is switched so that ergonomics of the wearable impact all conditions evenly. The participants were asked to follow the courses based on the game UI for the condition's display modality.

\section{DATA ANALYSES}

The XML data logs, generated from the PhotoNav game, were processed to determine each participant's time to reach each waypoint. From this, we calculated the average course traversal speed of each player (defined as the course distance divided by course completion time). Additionally, each player's location data were compared to the optimal path of the course (i.e., an optimal path was generated by using shortest distance between each waypoint, shown in Figure 5. Root-mean-square distance (RMSD) error was computed to investigate how much participants deviated from the optimal path. We calculated the percentage of time the UI was active during course traversal by each participant. Questionnaire data were compiled for statistical analyses.

\section{Statistical Analyses}

The independent variable was display modality and was manipulated within-subjects. The dependent variable was average course traversal speed, rather than completion time, since the latter was confounded by course length and difficulty. RMSD and the percentage of time participants actively used the PhotoNav interface were included in the analyses as covariates. Rather than a traditional analysis of variance (ANOVA), a linear mixed-model analysis was employed due to the presence of time-varying covariates in the design and partial data loss from one participant. While linear-mixed model techniques are relatively new, they are equally effective and more robust than traditional ANOVAs [21]. The complete linear-mixed model contained a single fixed factor, display modality, and the two aforementioned covariates. In addition, we used nonparametric Chi-square tests (Friedman and Wilcoxon signedrank) to examine responses to the questionnaire.

All participants completed the courses successfully using each interface. Movement data were successfully recorded from 11 of the 12 participants, with partial data loss for the final participant (values for RMSD and interface usage were unavailable in the handheld condition only; other values were captured). The data loss was minor and discovered late. Our choice of

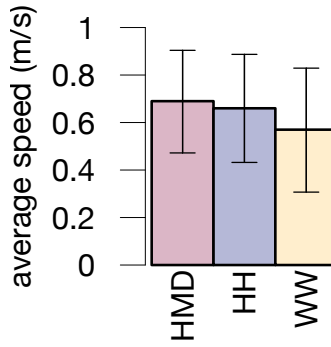

course $A$

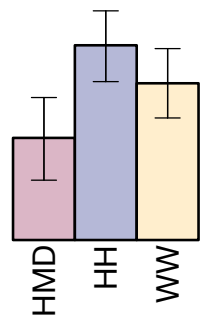

course B

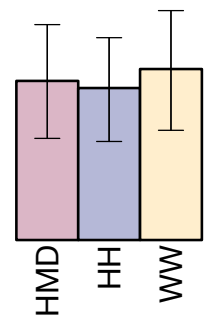

course $\mathrm{C}$
Figure 6. Estimated marginal means of player speed on each course with each display modality, accounting for covariates; error bars denote $95 \%$ confidence interval. (Note that $\mathrm{HH}$ is handheld and $\mathrm{WW}$ is wrist-worn.)

linear mixed models analysis is robust to missing data and allowed us to retain this participant in our analyses.

\section{RESULTS}

\section{Movement Data}

A visualization of the actual paths taken by players compared to the optimal paths appears in Figure 5. For Course B, the analysis revealed a significant effect of display modality, $[F(2,5.58)=6.44, \mathrm{p}=.035]^{4}$. Sidak-adjusted pairwise comparisons showed that participants traversed the course significantly faster using the handheld display $(M=.82)$ when compared with the HMD $(M=.43)$; performance for the wrist-worn display $(M=.66)$ did not differ from the others. However, there was no effect of display modality in Course A $[F(2,4.1)=.30, p=.76]$ nor in Course $\mathrm{C}[F(2,5.28)=.17$, $p=.85]$ (Figure 6). RMSD and the percentage of time that participants actively utilized the UI did not covary significantly with average course traversal speed (A RMSD: $[F(1,4.53)=3.74, p=.12]$, A UI use: $[F(1,5.38)=2.24$, $p=.19]$; B RMSD: $[F(1,5.47)=1.34, p=.30]$, B UI use: $[F(1,3.69)=1.86, p=.25]$; C RMSD: $[F(1,5.78)=1.20$, $p=.32]$, C UI use: $[F(1,6.94)=2.08, p=.19])$.

\section{Questionnaire Results}

We conducted Friedman and Wilcoxon signed-ranked (Chisquare) tests on participants questionnaire responses (questions described previously): ease of viewing clues, sense of safety, ease of viewing, and ease of matching. The sole independent variable was display modality. Figure 7 shows participant's rankings of different display modalities.

The Friedman test revealed significant differences in participants' rankings of the ease of viewing clues with the three interface modalities $\left[\chi^{2}(2)=6.62, p=.037\right]$. Wilcoxon signed-rank tests on each pair of modalities revealed significant differences between rankings of the $\operatorname{HMD}(M=2.75)$ and wrist-worn $(M=3.92)$ modalities [ $z=-2.14, p=.032]$, and marginal differences in rankings between $\operatorname{HMD}(M=2.75)$ and handheld $(M=3.92)$ modalities $[z=-1.85, p=.064]$. No difference in participants' rankings was observed between the handheld and wrist-worn modalities $[z=0]$.

Similarly, there was a significant effect of display modality $\left[\chi^{2}(2)=7.00, p=.030\right]$ for participants' perceived safety

\footnotetext{
${ }^{4}$ Note that fractional degrees of freedom are standard in linear mixedmodel analyses [43].
} 


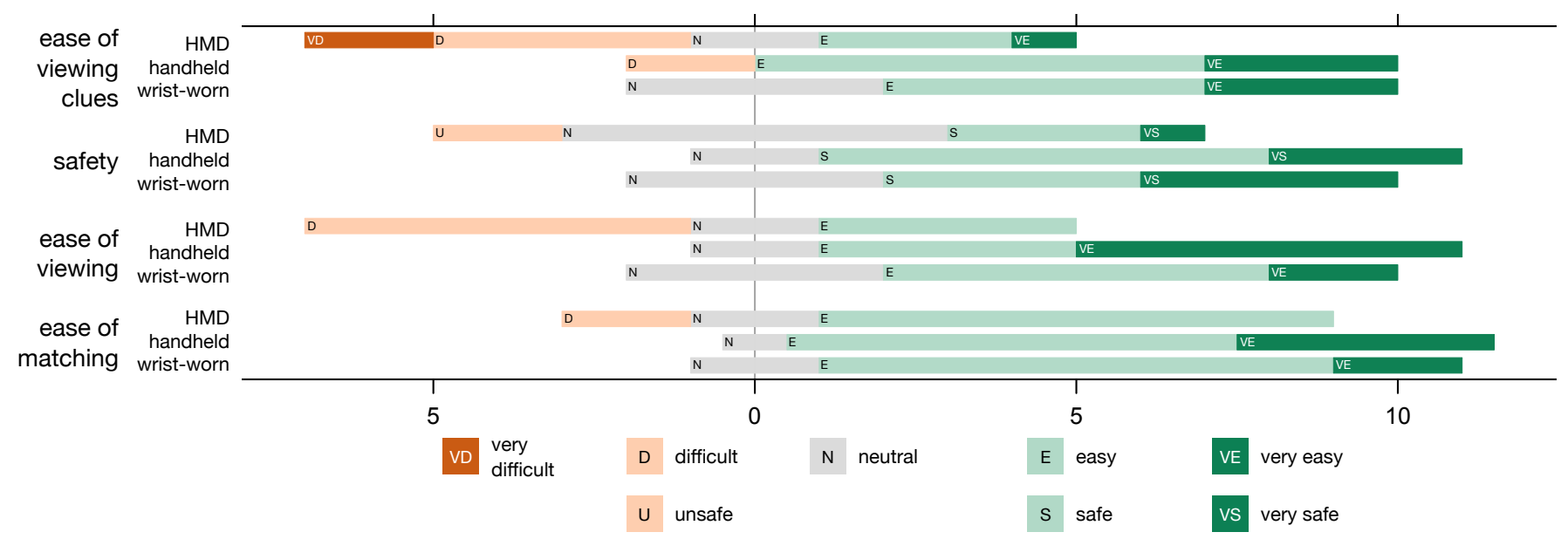

Figure 7. Participants' rankings of display modalities for ease of viewing hints, safety, ease of viewing in general, and ease of matching with the environment. $\mathrm{X}$-axis is the counts of each response by participants. Results indicate that the HMD is not favored by participants.

while navigating the courses using PhotoNav. Wilcoxon signed-rank tests on each pair of modalities revealed significant differences between rankings of the $\operatorname{HMD}(M=3.50)$ and handheld $(M=4.10)$ modalities $[z=-2.33, p=.020]$, and marginal differences in rankings between $\operatorname{HMD}(M=3.50)$ and wrist-worn $(M=4.00)$ modalities [ $z=-1.73, p=.084]$. No difference in participants' rankings was observed between the hand-held and wrist-worn modalities $[z=.38, p=.705]$.

For ease of viewing, the effect of display modality was significant $\left[\chi^{2}(2)=6.54, p=.038\right]$. Wilcoxon signed-rank tests on each pair of modalities revealed significant differences between rankings of the HMD $(M=2.83)$ and handheld $(M=4.33)$ modalities $[z=-2.44, p=.015]$, and significant differences in rankings between HMD $(M=2.83)$ and wristworn $(M=3.83)$ modalities $[z=-2.18, p=.029]$. There was a marginal difference in participants' rankings between the hand-held $(M=4.33)$ and wrist-worn $(M=3.83)$ modalities $[z=-1.90, p=.058]$.

Likewise, for ease of matching PhotoNav images with the outside world, the effect of display modality was significant $\left[\chi^{2}(2)=6.74, p=.034\right]$. Wilcoxon signed-rank tests on each pair of modalities revealed significant differences between rankings of the HMD $(M=3.50)$ and handheld $(M=4.25)$ modalities $[z=-2.07, p=.038]$, and marginal differences in rankings between handheld $(M=4.25)$ and wrist-worn $(M=4.00)$ modalities [ $z=-1.73, p=.083]$. No difference in participants' rankings was observed between the HMD and wrist-worn modalities $[z=-1.511, p=.131]$.

Additionally, we asked each participant what display modality they would prefer for outdoor navigation, 7 preferred handheld, 3 preferred wrist-worn, and 2 preferred head-mounted display. In response to which UI elements the participants focused on, to which participants could respond with multiple answers, we found that the hint photographic clue was mentioned 10 times; the current progress indicator 4 times; the overall progress indicator 4 times; and the destination photographic hint 1 time. None mentioned the time indicator.
For game enjoyment, across 36 ratings (12 participants $\times 3$ courses) no participants indicated that the game was "unenjoyable", 3 indicated "somewhat unenjoyable", and 2 were "neutral"; of the remaining 31: 14 considered the game "enjoyable" and 17 "very enjoyable".

\section{Course Familiarity}

Across all participants and courses, the familiarity button was only used twice. After completing each course, participants were asked about their familiarity with the course. Across 36 ratings (12 participants $\times 3$ courses) participants never indicated that they were "very familiar" or "familiar", 14 were "neutral", 10 were "unfamiliar" and 12 were "very unfamiliar".

\section{DISCUSSION}

In our study, we found that participants preferred using handheld or wrist-worn displays, but we also know that this is what participants are familiar with using in their everyday lives. It might be possible that features of our particular courses might have impacted the results. The study provided insights into the design of interfaces for wearables and mixed reality systems.

\section{Analyzing Game Design}

Overall, the data suggest the design of PhotoNav is successful. Participants' lack of familiarity with the courses suggests that course designs were effective, though an alternative explanation is that the workload of indicating familiarity was too high. Lack of familiarity indicates that participants would be reliant on the game, rather than their prior experience, to traverse the environment. Further, most participants paid attention to the hint view, which is the part of the UI we expect them to use to play. Participants reported that PhotoNav was fun.

\section{Issues with Head-Mounted Displays}

Our data suggest that there remain challenges for using HMDs in the physical world. In the present research, we observed issues with ambient light (e.g., sunlight) and challenges in merging the visual field. The HMD conditions performed the poorest, and were least preferred by participants. 
The prior research in this space aligns with our findings. The study conducted by Vadas et al. [47] found that the HMD's walking performance was worst. Similarly, Yeh et al. [52] observed that handheld displays performed better than HMDs. Our prior pilot study [38] of PhotoNav also showed that handheld performed better than HMD.

\section{Ambient Light}

Due to the use of a see-through HMD, one of the difficulties of outdoor experiments was found to be ambient light and glare caused by the sun. We observed that participants used their hands to block the ambient light while using the HMD so they could view the interface clearly. This aligns with a prior finding on the ARQuake game [44] that points out that bright sunlight makes it difficult to differentiate some colors from natural surroundings. Kerr et al. [19] also point out difficulty in viewing display because of bright sunlight. Since our game involves comparing images, some parts of the photographic clues might not have been clearly visible to participants in bright sunlight. Even though the HMD had a polarizing light filter to cut down on glare, and our prototyping experiments found it to be the best available with regards to sunlight, participants found it difficult to match images or see the UI.

Four out of 12 participants indicated that bright lights made it difficult to view images while using HMDs. P4 mentioned:

...HMD screen is transparent, which makes it difficult to see the hints under bright lights. I would suggest the screen to be made less transparent.

\section{Challenges in Merging the Visual Field}

When using the HMD, some participants mentioned that they found it difficult to match images because it was challenging to merge their visual field with the display. P11 mentioned:

For HMD, it was hard for me to concentrate on the system image while the view of the physical surrounding were presented on the background.

In the case of handheld or wrist-worn displays, the display is opaque and users can view information without distraction. Some studies have tried to provide recommendations on interface design to maximize visual recognition of interface elements. A study conducted by Gabbard et al. [24], studied six background textures commonly found in outdoor environments and six drawing styles and provide recommendations on colour choices and drawing style. Renkewitz et al. [35] conclude that colour and size of fonts used in HMDs should vary according to background, and they provide recommendations on optimal font size. Peterson et al. [35] found that selection time during visual search task is reduced by distributing overlapping labels in depth.

\section{Context of Use}

The PhotoNav UI was held constant across display modalities to avoid introducing bias, yet, Vogl et al. [48] concluded that interface design should be based on device and use context. Yeh et al. [52] found that clutter on HMDs rendered it less effective than handhelds with similar information. When we take this with the data on the weakness of the HMD in PhotoNav, it suggests that handheld or wrist-worn devices can be designed to provide more complex information (e.g., the norm for smartphones), while HMD use cases should minimize information and use micro-interactions (see next section).

In the conducted experiment, there were significant differences among the display modalities solely when participants traversed Course B. Courses A and C had many open areas, so players' freedom within the game rules resulted in larger number of decisions about where to go. Course B primarily ran in open spaces between buildings (not close enough to interfere with GPS), thus, players needed to make fewer decisions in the movement core mechanic. In addition, since participants were not provided a tutorial session, we expect that they acclimated to PhotoNav during Course A, where participants were getting used to the game interface and the nature of the mixed reality game. The pre-questionnaire showed that none of the participants had played mixed reality games before.

\section{Device Familiarity}

Our data suggest that participants' prior device experience biases their experience of display modality. We recorded display familiarity in pre-questionnaire and participants were not familiar with HMDs or wrist-worn displays; but were familiar with handheld displays. At the same time, handheld displays performed the best and were preferred by participants. Three participants said that they preferred the handheld device due to habitual use of a smartphone; P7 mentioned:

I was comfortable using the handheld display during the game because it was familiar to a smartphone and it was easy to handle it using my hand without putting pressure on any other body part like my wrist or head.

Despite the HMD offering the ability to see a player's surroundings, the familiarity of the handheld devices enables adept use by participants that they consider safe.

\section{Social Acceptance}

An informal observation was that participants were concerned about social acceptance of the wearable computer. Some participants preferred not to play the game during weekdays because of pedestrian crowd. They did not feel comfortable wearing the HMD in a crowded area. Profita et al. [34] surveyed 1,200 participants to analyze social acceptability of HMDs in a public setting. They examined how participant's judgment on the use of HMDs changed when information about HMD user's disability was made known. Their findings reveal that if the device was being used to support a person with a disability, HMD use was more socially acceptable.

\section{DESIGN IMPLICATIONS}

We derive design implications for wearable computers supporting mixed reality games based on our data with PhotoNav.

\section{Display Opacity}

See-through HMDs enable users to observe the physical environment; this is assumed to support situation awareness. Beyond issues with ambient light, problems with merging the visual field, especially with monocular displays, might pose 
a distraction while viewing information on the HMD. In fact, our participants found that the HMD inhibited their sense of safety, suggesting that the HMD did not support environment situation awareness.

One design choice might be to develop a modal display that can be almost opaque when information is displayed and seethrough when it is not. This would enable users to focus on the display for information, and maintain situation awareness when focus is elsewhere. A study by Laramee and Ware [22] also suggested that monocular transparent HMDs are not very useful in dynamically changing environments. We thus argue for the value of opaque displays, at least for monocular HMDs when there is a need to display complex information on the HMD screen. Future iterations of the PhotoNav experiment will test see-through and opaque displays.

\section{Detail Depends on Modality}

Our data, taken with prior work, suggest that information detail should depend heavily on display modality. We expect that HMD use cases should focus on micro-interactions, events that take place briefly (i.e., four seconds or less) [2], rather than interfaces with prolonged interaction. Micro-interactions should be based on screen elements that are easy to spot on the HMD, with minimal detail. A study conducted by Vogl et al. [48] on everyday use of head-worn computers also suggested that HMDs should be used for micro-interactions. Further bolstering this point is an earlier study by Oulasvirta et al. [33], which showed users' attention span for a mobile device is about $4-8$ seconds.

Since many outdoor use cases of display modalities require users to split their attention, we suggest that in case of viewing tasks, UIs should be designed for micro-interactions. We suggest that handheld displays can still have complex interactions compared to HMDs since handhelds provide less distractions as UI is not merged with the environment. Further experiments are needed to determine how details in interfaces should be adapted based on display modalities.

\section{Static Interfaces}

The PhotoNav interface is an information-display interface: players did not need to navigate the UI for further information. Our questionnaire results suggest that players focused mostly on the hint photographic clue and the current progress indicator. The results suggest that immediate contextual information is crucial for tasks requiring split attention.

\section{Affordances and Constraints of Physical-World Spaces}

Norman considers how artifacts enable action through affordances and prevent action through constraints [32]; these properties apply to physical-world environments as well. We advise designers of mixed reality games to consider the physical world context of play not just as found objects [23] that afford physical gameplay or facilitate safety, but as an essential object, to which the player always attends, leverages, and engages with, as a component of play. For example, many photographic clues did not contain images that showed names of buildings, this was done to introduce more challenge to the players by utilizing nature of the environment.
MacIntyre identifies aura of places as the intangible significance of a physical location [25], which has implications for the narratives one might develop in a space. In prior systems (e.g., $[13,51])$ location becomes vital part of mixed reality experience that connects participants to a narrative.

We observe that weather plays a big part in scheduling a mixed reality game, as many times the game session had to be canceled prior to the start because of cold weather. The game had to be run during the daytime so this limited gameplay schedule. Standard digital games that can be played in PCs, consoles or mobile phone do not come with constraints of weather, sunlight or daylight, the nature of outdoor mixed reality games make it challenging to reach a mass audience.

\section{CONCLUSION}

In this paper, we described a mixed reality game, PhotoNav, in which participants navigate the physical world, guided by photographic clues. The game design is aimed at investigating the architecture of wearable computers, specifically selecting the best display modality for navigation. Furthermore, we presented the results of a user study, in which participants played PhotoNav while traversing different courses using three distinctive display modalities.

Our results showed that handheld displays performed better in one of the courses and users preferred handheld as well. While we expected the HMD to outperform other modalities, we discovered the opposite. Factors that might have resulted in players poor performance included ambient sunlight and participants' inability to discern information displayed on the interface. Lack of familiarity with the display modality also likely contributed to the HMD's failure.

Our data, synthesized with the literature, suggest a number of design implications for mixed reality systems. The seethrough nature of some HMDs inhibits readability, which suggests that such displays should be opaque when data is displayed. The level of detail that should be used in a UI depends on the display modality: HMDs are best suited to lowdetail information that changes infrequently. Finally, spaces afford and constrain action, and physical space should be deeply incorporated into the design of mixed reality games.

Open questions remain about how UIs can best adapt, in terms of design and detail, to match context of use and type of device. As new and improved HMDs become more mainstream, investigation of HMDs that adapt their transparency according to ambient light and root their design on micro-interactions might be useful. In light of the UIs' context dependency and display modalities, accurate comparison of such modalities while optimizing their design remains a big challenge.

\section{ACKNOWLEDGMENTS}

We thank all of our research subjects. Author Sharma wishes to thank Sultan A. Alharthi for helping with video development. Author Toups wishes to thank NMSU for its financial support of his team. This material is based upon work supported by the National Science Foundation under Grant Nos. IIS-0803854, IIS-0742947. 


\section{REFERENCES}

1. Daniel Ashbrook, Kent Lyons, James Clawson, and Thad Starner. 2009. Smart Clothing: Technology and Applications. CRC Press, Chapter Methods of Evaluation for Wearable Computing, 229-248.

2. Daniel Lee Ashbrook. 2010. Enabling Mobile Microinteractions. Ph.D. Dissertation. Massachusetts Institute of Technology.

3. Ronald T. Azuma. 1999. The Challenge of Making Augmented Reality Work Outdoors. In Mixed Reality: Merging Real and Virtual. Springer-Verlag, 379-390.

4. Ashweeni Beeharee and Anthony Steed. 2007. Minimising pedestrian navigational ambiguities through geoannotation and temporal tagging. In Human-Computer Interaction. Interaction Platforms and Techniques. Springer, 748-757.

5. Ashweeni Kumar Beeharee and Anthony Steed. 2006. A Natural Wayfinding Exploiting Photos in Pedestrian Navigation Systems. In Proceedings of the 8th Conference on Human-computer Interaction with Mobile Devices and Services (MobileHCI '06). ACM, New York, NY, USA, 81-88. DOI :

http://dx.doi.org/10.1145/1152215.1152233

6. Steve Benford, Andy Crabtree, Martin Flintham, Adam Drozd, Rob Anastasi, Mark Paxton, Nick Tandavanitj, Matt Adams, and Ju Row-Farr. 2006. Can you see me now? ACM Transactions on Computer-Human Interaction 13, 1 (2006), 100-133. DOI: http://dx.doi.org/10.1145/1143518.1143522

7. Steve Benford and Gabriella Giannachi. 2011. Performing Mixed Reality. MIT Press.

8. Olav W. Bertelsen and Christina Nielsen. 2000. Augmented Reality As a Design Tool for Mobile Interfaces. In Proceedings of the 3rd Conference on Designing Interactive Systems: Processes, Practices, Methods, and Techniques (DIS '00). ACM, New York, NY, USA, 185-192. DOI:

http://dx . doi .org/10.1145/347642 . 347714

9. Elizabeth M. Bonsignore, Derek L. Hansen, Zachary O. Toups, Lennart E. Nacke, Anastasia Salter, and Wayne Lutters. 2012. Mixed reality games. In Proceedings of the ACM 2012 conference on Computer Supported Cooperative Work Companion (CSCW'12). ACM, New York, NY, USA, 7-8. DOI : http://dx.doi.org/10.1145/2141512.2141517

10. Gaetano Borriello, Matthew Chalmers, Anthony LaMarca, and Paddy Nixon. 2005. Delivering Real-world Ubiquitous Location Systems. Commun. ACM 48, 3 (March 2005), 36-41. DOI : http://dx.doi.org/10.1145/1047671.1047701

11. Bo Dahlbom and E. Ljungberg. 1998. Mobile informatics. Scandinavian Journal of Information Systems 10 (1998), 227-234.
12. Paul Dourish. 2001. Where the Action Is: The Foundations of Embodied Interaction (1st ed.). MIT Press, Cambridge, MA, USA.

13. Steven Dow, Jaemin Lee, Christopher Oezbek, Blair Maclntyre, Jay David Bolter, and Maribeth Gandy. 2005. Exploring Spatial Narratives and Mixed Reality Experiences in Oakland Cemetery. In Proceedings of the 2005 ACM SIGCHI International Conference on Advances in Computer Entertainment Technology (ACE '05). ACM, New York, NY, USA, 51-60. DOI : http://dx.doi.org/10.1145/1178477.1178484

14. Steven Fadden, Patricia May Ververs, and Christopher D. Wickens. 1998. Costs and benefits of head-up display use: A meta-analytic approach. In Proceedings of the Human Factors and Ergonomics Society Annual Meeting 1998, Vol. 42. 16-20.

15. Steven Feiner, Blair MacIntyre, Tobias Hollerer, and Anthony Webster. 1997. A Touring Machine: Prototyping 3D Mobile Augmented Reality Systems for Exploring the Urban Environment. In ISWC '97: Proceedings of the 1st IEEE International Symposium on Wearable Computers. IEEE Computer Society, 208-217.

16. Jeffrey Hightower and Gaetano Borriello. 2001. Location Systems for Ubiquitous Computing. Computer 34, 8 (2001), 57-66. DOI : http://dx.doi .org/10.1109/2.940014

17. Andruid Kerne, Yin Qu, Andrew M. Webb, Sashikanth Damaraju, Nic Lupfer, and Abhinav Mathur. 2010. Meta-metadata: A Metadata Semantics Language for Collection Representation Applications. In Proceedings of the 19th ACM International Conference on Information and Knowledge Management (CIKM '10). ACM, New York, NY, USA, 1129-1138. DOI : http://dx. doi .org/10.1145/1871437.1871580

18. Andruid Kerne, Zachary O. Toups, Blake Dworaczyk, and Madhur Khandelwal. 2008. A Concise XML Binding Framework Facilitates Practical Object-oriented Document Engineering. In Proceedings of the Eighth ACM Symposium on Document Engineering (DocEng '08). ACM, New York, NY, USA, 62-65. DOI: http://dx.doi.org/10.1145/1410140.1410152

19. Steven J. Kerr, Mark D. Rice, Yinquan Teo, Marcus Wan, Yian Ling Cheong, Jamie Ng, Lillian Ng-Thamrin, Thant Thura-Myo, and Dominic Wren. 2011. Wearable Mobile Augmented Reality: Evaluating Outdoor User Experience. In Proceedings of the 10th International Conference on Virtual Reality Continuum and Its Applications in Industry (VRCAI '11). ACM, New York, NY, USA, 209-216. DOI : http://dx.doi .org/10.1145/2087756.2087786

20. Steinar Kristoffersen and Fredrik Ljungberg. 1998. Representing modalities in mobile computing. Proceedings of Interactive applications of mobile computing (1998). 
21. Charlene Krueger and Lili Tian. 2004. A Comparison of the General Linear Mixed Model and Repeated Measures ANOVA Using a Dataset with Multiple Missing Data Points. Biological Research For Nursing 6, 2 (2004), 151-157. DOI : http://dx.doi.org/10.1177/1099800404267682

22. Robert S. Laramee and Colin Ware. 2002. Rivalry and Interference with a Head-mounted Display. ACM Trans. Comput.-Hum. Interact. 9, 3 (Sept. 2002), 238-251. DOI : http://dx.doi.org/10.1145/568513.568516

23. Lucy R. Lippard. 1971. Marcel Duchamp. In Dadas on Art: Tzara, Arp, Duchamp, and Others, Lucy R. Lippard (Ed.). Dover Publications, Inc., 139-154.

24. Mark A. Livingston, Joseph L. Gabbard, J. Edward Swan II, Ciara M. Sibley, and Jane H. Barrow. 2013. Basic perception in head-worn augmented reality displays. In Human factors in augmented reality environments. Springer, 35-65.

25. Blair MacIntyre, Jay David Bolter, and Maribeth Gandy. 2004. Presence and aura of meaningful places. In 7th Annual International Workshop on Presence (PRESENCE 2004). 36-43.

26. Steve Mann. 1997. Wearable computing: A first step toward personal imaging. Computer 30, 2 (1997), 25-32.

27. Steve Mann, Jason Nolan, and Barry Wellman. 2003. Sousveillance: Inventing and using wearable computing devices for data collection in surveillance environments. Surveillance \& Society 1, 3 (2003), 331-355.

28. Andrew J. May, Tracy Ross, Steven H Bayer, and Mikko J. Tarkiainen. 2003. Pedestrian navigation aids: Information requirements and design implications. Personal and Ubiquitous Computing 7, 6 (2003), 331-338.

29. Paul Milgram and Fumio Kishino. 1994. A taxonomy of mixed reality visual displays. IEICE Transactions on Information and Systems E77-D, 12 (1994), 1321-1329.

30. Keith Mitchell, Duncan McCaffery, George Metaxas, Joe Finney, Stefan Schmid, and Andrew Scott. 2003. Six in the city: Introducing Real Tournament - a mobile IPv6 based context-aware multiplayer game. In NETGAMES '03: Proceedings of the 2nd Workshop on Network and System Support for Games. ACM Press, 91-100. D0I : http://dx.doi.org/10.1145/963900.963909

31. Stefan Münzer, Hubert D. Zimmer, Maximilian Schwalm, Jörg Baus, and Ilhan Aslan. 2006. Computer-assisted navigation and the acquisition of route and survey knowledge. Journal of Environmental Psychology 26, 4 (2006), 300-308. DOI :

http://dx.doi.org/10.1016/j.jenvp.2006.08.001

32. Don Norman. 2013. The Design of Everyday Things: Revised and Expanded Edition (revised 2013 ed.). Basic Books.

33. Antti Oulasvirta, Sakari Tamminen, Virpi Roto, and Jaana Kuorelahti. 2005. Interaction in 4-second Bursts: The
Fragmented Nature of Attentional Resources in Mobile HCI. In Proceedings of the SIGCHI Conference on Human Factors in Computing Systems (CHI '05). ACM, New York, NY, USA, 919-928. DOI :

http://dx.doi.org/10.1145/1054972.1055101

34. Halley Profita, Reem Albaghli, Leah Findlater, Paul Jaeger, and Shaun K. Kane. 2016. The AT Effect: How Disability Affects the Perceived Social Acceptability of Head-Mounted Display Use. In Proceedings of the 2016 CHI Conference on Human Factors in Computing Systems (CHI '16). ACM, New York, NY, USA, 4884-4895. DOI :

http://dx.doi.org/10.1145/2858036.2858130

35. Helge Renkewitz, Verena Kinder, Mario Brandt, and Thomas Alexander. 2008. Optimal font size for head-mounted-displays in outdoor applications. In Information Visualisation, 2008. IV'08. 12th International Conference. IEEE, 503-508.

36. Katie Salen and Eric Zimmerman. 2004. Rules of Play: Game Design Fundamentals. MIT Press, Cambridge, MA, USA.

37. Beatriz Sousa Santos, Paulo Dias, Angela Pimentel, Jan-Willem Baggerman, Carlos Ferreira, Samuel Silva, and Joaquim Madeira. 2009. Head-mounted display versus desktop for 3D navigation in virtual reality: A user study. Multimedia Tools and Applications 41, 1 (2009), 161-181. http://dx.doi.org/10.1007/s11042-008-0223-2

38. Hitesh Nidhi Sharma, Zachary O. Toups, Ajit Jain, and Andruid Kerne. 2015. Designing to Split Attention in a Mixed Reality Game. In Proceedings of the 2015 Annual Symposium on Computer-Human Interaction in Play (CHI PLAY '15). ACM, New York, NY, USA, 691-696. DOI : http://dx.doi.org/10.1145/2793107.2810289

39. Sarah Sharples, Sue Cobb, Amanda Moody, and John R. Wilson. 2008. Virtual reality induced symptoms and effects (VRISE): Comparison of head mounted display (HMD), desktop and projection display systems. Displays 29, 2 (2008), 58-69.

40. Thad Starner, Steve Mann, Bradley Rhodes, Jeffery Levine, Jennifer Healey, Dana Kirsch, Roz Picard, and Alex Pentland. 1997. Augmented Reality Through Wearable Computing. Presence 6, 4 (Winter 1997), 386-398.

41. Thad Eugene Starner. 1999. Wearable computing and contextual awareness. Ph.D. Dissertation. Massachusetts Institute of Technology.

http://dx.doi.org/10.1162/pres.1997.6.4.386

42. Colin Swindells, Barry A. Po, Ima Hajshirmohammadi, Brian Corrie, John C. Dill, Brian D. Fisher, and Kellogg S. Booth. 2004. Comparing CAVE, wall, and desktop displays for navigation and wayfinding in complex 3D models. In Proceedings of the Computer Graphics International. IEEE Computer Society, 420-427. DOI :

http://dx.doi.org/10.1109/CGI.2004.1309243 
43. Kunio Takezawa. 2014. Learning Regression Analysis by Simulation. Springer Japan, Tokyo, Chapter Linear Mixed Model, 269-294. DOI :

http://dx.doi.org/10.1007/978-4-431-54321-3_6

44. Bruce Thomas, Ben Close, John Donoghue, John Squires, Phillip De Bondi, Michael Morris, and Wayne Piekarski. 2000. ARQuake: An outdoor/indoor augmented reality first person application. In Wearable Computers, The Fourth International Symposium on. 139-146. DOI : http://dx.doi.org/10.1109/ISWC. 2000.888480

45. Bruce Thomas, Ben Close, John Donoghue, John Squires, Phillip De Bondi, and Wayne Piekarski. 2002. First Person Indoor/Outdoor Augmented Reality Application: ARQuake. Personal Ubiquitous Computing 6, 1 (Jan. 2002), 75-86. DOI :

http://dx.doi.org/10.1007/s007790200007

46. Daniela G. Trevisan, Monica Gemo, Jean Vanderdonckt, and Benoît Macq. 2004. Focus-based Design of Mixed Reality Systems. In Proceedings of the 3rd Annual Conference on Task Models and Diagrams (TAMODIA '04). ACM, New York, NY, USA, 59-66. DOI : http://dx.doi.org/10.1145/1045446.1045458

47. Kristin Vadas, Kent Lyons, Daniel Ashbrook, Ji Soo Yi, Thad Starner, and Julie Jacko. 2006. Reading on the Go: An Evaluation of Three Mobile Display Technologies. Technical Report GIT-GVU-06-09. Georgia Institute of Technology.
48. Anita Vogl, Nicolas Louveton, Rod McCall, Mark Billinghurst, and Michael Haller. 2015. Understanding the everyday use of head-worn computers. In Human System Interactions (HSI), 2015 8th International Conference on. IEEE, 213-219.

49. Christopher D. Wickens. 1997. Attentional issues in head-up displays. Engineering psychology and cognitive ergonomics: 1 (1997), 3-21.

50. Christopher D. Wickens and Jeffry Long. 1995. Object versus space-based models of visual attention: Implications for the design of head-up displays. Journal of Experimental Psychology: Applied 1, 3 (1995), 179.

51. Jason Wither, Rebecca Allen, Vids Samanta, Juha Hemanus, Yun-Ta Tsai, Ronald Azuma, Will Carter, Rachel Hinman, and Thommen Korah. 2010. The westwood experience: connecting story to locations via mixed reality. In 2010 IEEE International Symposium On Mixed and Augmented Reality-Arts, Media, and Humanities (ISMAR-AMH). IEEE, 39-46.

52. Michelle Yeh, James L. Merlo, Christopher D. Wickens, and David L. Brandenburg. 2003. Head up versus head down: The costs of imprecision, unreliability, and visual clutter on cue effectiveness for display signaling. Human Factors: The Journal of the Human Factors and Ergonomics Society 45, 3 (2003), 390-407.

53. Jean-Marie Zogg. 2007. Essentials of Satellite Navigation - Compendium. Technical Report GPS-X-02007-C. u-blox AG. 